\title{
Bases sociais das emoções do consumidor - uma abordagem complementar sobre emoções e consumo
}

\author{
Social bases of consumer emotions - a complementary approach to emotions \\ and consumption
}

\author{
João Felipe Rammelt Sauerbronn ${ }^{1}$ \\ Eduardo André Teixeira Ayrosa \\ Denise Franca Barros ${ }^{3}$
}

Resumo

O estudo das emoções no comportamento de compra e consumo dos indivíduos ganhou relevância nas últimas duas décadas, inclusive, no Brasil. A maior parte dos estudos, no entanto, parece oferecer uma única perspectiva para o estudo do fenômeno: aquela advinda da psicologia cognitiva. Apesar da reconhecida importância dessa visão, acreditamos que a adoção de novas perspectivas no estudo das emoções no consumo pode contribuir positivamente para a maior compreensão do tema. Além de oferecer contribuições para a prática do marketing, o estudo das emoções relacionadas ao consumo deve também apontar direções para a ampliação das bases filosóficas da disciplina, bem como, novas possibilidades à pesquisa gerencial de mercado. Neste estudo, observamos o estudo das emoções na pesquisa acadêmica de marketing recente, especialmente, no Brasil, discutindo as diferentes perspectivas existentes. A sugestão de uma abordagem complementar, baseada nos pressupostos da sociologia das emoções, e as implicações metodológicas dessa proposição encerram o artigo.

Palavras-chave: consumo; emoções; sociologia das emoções.

\section{Abstract}

The focus of the present paper is on the sociological aspects of consumption emotions and how to develop an alternative approach to the study of emotions related to consumption. The study of emotions involved in buying and consuming behavior has grown in relevance in the past two decades among consumer researchers. Prior work on consumption emotions has emphasized the cognitive psychology's perspective. We believe that the introduction of new perspectives on the study of consumption emotions can contribute positively for a better understanding of the theme. We explore how other authors, including Brazilian ones, deal with the consumption emotions on their works and discuss different perspectives adopted. We suggest a complementary approach to the consumption emotions based on a new field of sociology: the sociology of emotions. At the end, we discuss the methodological implications of this approach.

Key words: consumption; emotions; sociology of emotions.

\footnotetext{
${ }^{1}$ Doutor em Administração pela Escola Brasileira de Administração Pública e de Empresas- EBAPE/FGV-RJ; Mestre em Administração Pública pela EBAPE/FGV-RJ; Bacharel em Ciências Econômicas pelo IE/UFRJ. Professor Adjunto do Programa de Mestrado em Administração da Escola de Gestão de Negócios da Universidade Unigranrio. Endereço: Rua da Lapa, 86 - 90 andar - Lapa - Rio de Janeiro - Brasil - CEP: 20021-180. E-mail: joao.sauerbonn@fgv.br

${ }^{2}$ Doutor em Administração pela London Business School; Mestre em Administração pelo Instituto de Pesquisa e Pós-Graduação em Administração de Empresas da Universidade Federal do Rio de Janeiro - Coppead/UFRJ; Bacharel em Engenharia UFRJ. Professor Adjunto da Escola Brasileira de Administração Pública e de Empresas da Fundação Getulio Vargas - EBAPE/FGV. Endereço: Praia de Botafogo, 190 - sala 527 - Botafogo - Rio de Janeiro/RJ - Brasil - CEP: 22253-900. E-mail: eduardo.ayrosa@fgv.br

${ }^{3}$ Doutoranda da Escola Brasileira de Administração Pública e de Empresas da Fundação Getulio Vargas- EBAPE/FGV.ENDEREÇO: Praia de Botafogo, 190 sala 527 - Botafogo - Rio de Janeiro/RJ - Brasil - CEP: 22253-900. Email: denise.barros@fgv.br

Artigo submetido em abril e aceito em agosto de 2008.
} 


\section{Introdução}

O estudo das emoções no comportamento de compra e consumo dos indivíduos ganhou relevância nas últimas duas décadas. Pesquisadores de consumo com orientações diversas, como Holbrook e Hirschman (1982), Richins (1997), e Westbrook e Oliver (1991), apontam o papel ativo das emoções durante as experiências de consumo. Um considerável corpo de pesquisa reconheceu as emoções como um dos mais importantes fatores em respostas específicas de consumidores a estímulos de marketing e ao comportamento do consumidor em geral. Por exemplo, as respostas emocionais de consumidores foram o foco central de estudos a respeito do impacto da propaganda (HOLBROOK; BATRA, 1987), da formação de julgamentos de satisfação (WESTBROOK; OLIVER, 1991) e de processos de tomada de decisão (LUCE; PAYNE; BETTMAN, 1999). Como aponta Richins (1997, p.127), "a importância das emoções na esfera do comportamento do consumidor já está firmemente estabelecida".

Apesar do reconhecimento, ainda não há uma pesquisa sistemática sobre os determinantes das emoções e os seus efeitos nas respostas dos consumidores (AHUVIA, 2005). Bagozzi, Poinath e Nyer (1999) apontam para a necessidade de se oferecer uma explicação mais profunda da natureza complexa do processo e para a fenomenologia da resposta emocional associada ao consumo. Sentimentos como raiva, vergonha ou culpa foram analisados como respostas ou iniciadores do consumo. Tais estudos parecem acompanhar a idéia de que as emoções acompanham os indivíduos a todo o momento (FOURNIER, 1998) e que mesmo estados emocionais mais brandos podem afetar substancialmente o processo cognitivo e o comportamento social (AAKER; STAYMAN; HAGERTY, 1986).

A academia brasileira já demonstrou profundo interesse no tema e contribuiu para a discussão sobre as emoções no consumo. A produção nacional, no entanto, parece oferecer uma única perspectiva para o estudo do fenômeno, baseada fortemente no conhecimento advindo da psicologia cognitiva. Acreditamos que a pluralidade de olhares sobre o tema pode contribuir positivamente para o maior entendimento do tema. Por esse motivo, este artigo se dedica a discutir a proposição de uma abordagem complementar aos estudos sobre as emoções no consumo baseada na compreensão das bases sociais das emoções.

Este ensaio procura traçar um panorama da pesquisa acadêmica sobre emoções e consumo, especialmente, no Brasil, e discutir diferentes perspectivas existentes. Como resultado, são apresentadas uma abordagem complementar, baseada nos pressupostos da sociologia das emoções, bem como as implicações metodológicas dessa proposição.

\section{Antecedentes do estudo das emoções no consumo}

Historicamente, a psicologia se estabeleceu como local de discussão e pesquisa sobre as emoções, dada a primazia de interesse e o volume de trabalhos empíricos sobre emoções desenvolvidos dentro da área. Ocorre, porém, que mesmo no campo da psicologia ocorrem divergências sobre esse tema e as diferentes correntes apresentam definições diversas para o conceito. De uma forma geral, as emoções podem ser entendidas primordialmente como estados internos da pessoa que envolvem interações entre o sujeito e um objeto, tais como sentimentos, estados de satisfação ou ativação de determinado padrão motor (FRIJDA, 2004).

Esses estados vivenciados pelos indivíduos apresentam diferentes formas de expressão, e a discriminação desses estados de mobilização se dá a partir da definição de sentimentos (rótulos atribuídos a determinados estados emocionais). Os sentimentos são mais facilmente perceptíveis, enquanto as emoções, de maneira genérica, referem-se a qualquer estado interno da pessoa resultante da interação entre um sujeito e um objeto (FRIJDA, 2004). A susceptibilidade que o ser humano experimenta perante determinadas alterações do mundo externo ou em si próprio é o afeto, que pode ser positivo ou negativo (MOORE; FINE, 1992). 
A pesquisa que adota a abordagem da psicologia cognitiva é voltada para a predição de escolhas baseada em fluxos causais e é muito eficiente para resolver problemas de interesse mais direto dos praticantes, como escolhas de marca ou sensibilidade a apelos de propaganda. Há, contudo, aspectos importantes que permanecem sem explicação ou sem exame mais detalhado. Acreditamos que as emoções envolvidas com o consumo podem ser mais profundamente explicadas e o conhecimento produzido na área de marketing pode ser orientado por outros aspectos além dos interesses das unidades produtoras.

$\mathrm{O}$ ato de consumo é uma experiência humana produtora de significado que é muito difícil de ser reduzida à previsão, sob pena de se incorrer em alguma deficiência na observação do fenômeno. Os pesquisadores de consumo ainda não se comprometeram a desenvolver estudos capazes de ampliar o conhecimento sobre as formas com as quais o consumidor vivencia pensamentos, emoções, atividades e valores humanos. Para Moorman (1987), o consumo envolve essencialmente a satisfação de fantasias (estimuladas artificialmente ou não) e a concretização de uma fantasia alienada de nossos seres concretos. É, de fato, obrigação do pesquisador de consumo construir teorias sobre o consumo que reflitam a realidade em toda a sua complexidade (HOLBROOK, 1995). Dessa forma, os estudos a respeito das emoções no consumo não podem se extinguir, na medida em que os problemas dos praticantes naquele momento estejam resolvidos.

Seguindo a indicação de Bagozzi, Gürhan-Canli e Priester (2002), buscamos apresentar neste estudo, uma forma de se entender o fenômeno emocional no consumo a partir de suas bases sociais. Mesmo que a experiência da emoção seja privada ao indivíduo, suas causas partem de interpretações ou respostas advindas do grupo social no qual o indivíduo se insere. Apesar da vasta maioria da pesquisa em comportamento emocional apresentar um enfoque individualista (PARKINSON, 1995), as relações de marketing realizam-se em contextos onde a perspectiva social das emoções pode apresentar contribuições importantes sobre o conhecimento a respeito do comportamento dos consumidores. Acreditamos que esse objetivo seja relevante, não apenas para pesquisadores interessados em fazer incursões no terreno das emoções, mas também para praticantes, principalmente, na área da pesquisa gerencial de marketing, uma vez que emoções podem ter influência abrangente em atitudes e comportamentos dos consumidores.

Nossa proposta parte de um exame crítico das formas de uso das emoções na pesquisa acadêmica de marketing para, em seguida, apresentarmos uma abordagem complementar alternativa. Nossa crítica, portanto, não recai sobre a forma como o conceito é formulado, mas, sim, sobre a utilização exclusiva do ferramental da psicologia cognitiva e sobre como as formas mais usuais de tratamento das emoções são exploradas no campo do marketing.

Não procuramos retomar a discussão sobre a predominância do componente cognitivo no processo emocional, assunto amplamente discutido por Tsal (1985) e Lazarus (1982), de um lado, e por Zajonc (1980) e Markus (ZAJONC; MARKUS, 1982, 1985), de outro. No entanto, reconhecemos que o debate acerca da primazia da cognição foi importante, na medida em que recrutou pesquisadores interessados em uma abordagem alternativa à exclusividade cognitivista e chamou atenção para a possibilidade de interação entre cognição e afeto e, conseqüentemente, para outras formas de se entender o fenômeno emocional no consumo.

Também não é parte do escopo deste estudo fazer uma revisão exaustiva sobre emoção e consumo, mas, sim, rever como o tema tem sido usado por pesquisadores nas áreas de marketing e comportamento do consumidor, especialmente, no Brasil. Dessa forma, poderemos apresentar de forma mais consistente nosso ponto e trazer o leitor à compreensão e à discussão de um ponto de vista complementar alternativo ao estudo das emoções no consumo. Assim, a próxima seção discute a perspectiva cognitiva das emoções.

\section{A abordagem cognitivista no estudo das emoções no marketing}

A pesquisa de marketing tradicionalmente se preocupa com a geração de conhecimento para dar suporte às decisões de praticantes da área. Os estudos do comportamento dos consumidores podem revelar fatores determinantes para a compra e o consumo de produtos e serviços. Contudo, o foco desses estudos está nas 
decisões de compra e nas escolhas de marca, o que indica uma aparente primazia do domínio gerencial em detrimento do domínio filosófico do marketing (BENTON JR., 1987). Tal linha de atuação auxiliou a construção de uma reputação eminentemente instrumental do marketing, uma visão bastante restrita de seu campo.

Com o intuito de desenvolver estudos que fundamentassem a tomada de decisão gerencial, os pesquisadores de marketing encontraram na psicologia cognitiva, amplas referências de atuação, tendo desenvolvido, a partir de pressupostos dessa disciplina, sólidas bases para suas pesquisas. Comumente utilizada pelos pesquisadores da área, a figura do "homem-computador" é empregada para explicar como o fenômeno emocional interfere no processo de tomada de decisão do indivíduo (HIRSCHMAN; STERN, 1999). Estendendo tal visão ao estudo do comportamento do consumidor, o ato de consumo é visto como uma série de decisões racionais que se iniciam com o processamento de informações que possibilitam a escolha de um produto e culminam na decisão de compra.

Os modelos e teorias tradicionais de comportamento do consumidor assumem que um conjunto de eventos ou atividades ocorre quando um consumidor processa informação: a atividade cognitiva é seguida por avaliação afetiva que resulta em um comportamento, como nos modelos de Engel, Blackwell e Miniard (2000), de Fishbein e Azjen (1979) e de Howard e Sheth (apud HOWARD, 1969) e Oliver (1980). Todos esses modelos acabam por retratar o consumidor como um processador de informações isento de paixões e imerso em um mundo particular, só seu. Esses modelos são considerados capazes de predizer o comportamento dos consumidores, como afirmam Peterson, Hoyer e Wilson (1986), mesmo que as influências afetivas tenham sido significativamente subestimadas quando do desenho de pesquisa. Dessa forma, criam-se modelos efetivos dentro de uma perspectiva teórica válida segundo os pressupostos da abordagem dominante, mas que têm bases que limitam o conhecimento mais completo do fenômeno do consumo.

A noção de um fluxo causal que caminha da cognição ao afeto e ao comportamento, lançada por Howard (1969), domina a pesquisa sobre o comportamento dos consumidores e foi adotada por inúmeros pesquisadores da área. Engel, Blackwell e Miniard (2000) propõem uma cadeia que começa com o reconhecimento da necessidade do processamento de informações, segue com a avaliação de alternativas e finaliza com a compra. As emoções são consideradas exclusivamente como variáveis que auxiliam a compreensão das escolhas de consumo. Toda a complexidade e variedade do fenômeno emocional é encarada a partir de uma representação do afeto, concebida de forma muito restrita e que se dá no sentido: cognição - afeto - comportamento.

Diversos estudos encontraram formas de modelar a relação entre cognição, afeto e comportamento. Batra e Silk (1984) e Lutz, Mackenzie e Belch (1983) apresentaram respostas afetivas adicionais envolvendo as atitudes diante da propaganda como mediadoras interferentes entre a cognição e o comportamento do consumidor. Ahtola (1985) e Batra (1985) propuseram a distinção entre os componentes hedonistas e utilitaristas do afeto, operacionalizando uma dupla influência afetiva, submissa à capacidade cognitiva do indivíduo.

Influentes trabalhos sobre as emoções no consumo foram desenvolvidos por Westbrook e Oliver (OLIVER, 1980; WESTBROOK; OLIVER, 1991). A preocupação de Oliver está localizada no desafio de compreender a satisfação do consumidor, e, assim, a forma e o momento de observação do fenômeno emocional são bastante específicos. Os trabalhos de Westbrook e Oliver trazem, de imediato, referências da psicologia cognitiva de Izard (1997) e da psicologia evolutiva de Plutchik (1980), além de adotarem procedimentos metodológicos bastante tradicionais, partindo para a construção de medidas que possibilitem a proposição de taxonomias de emoções relacionadas à satisfação do consumidor, com base numa lista das emoções básicas. A preocupação metodológica dedicada ao rigor das medidas e à validade do construto denota a devoção desses autores ao desenvolvimento de instrumentos aplicáveis dentro do universo positivista da disciplina do marketing, assim como suas convicções ontológicas a respeito de predição e construção de "verdades" únicas.

Não é surpreendente notarmos que os trabalhos desenvolvidos a partir do referencial proposto por Oliver e Westbrook reproduzem suas preocupações metodológicas e epistemológicas orientadas para a confirmação do referencial teórico advindo da psicologia cognitiva. Trabalhos como o de Phillips e Baumgartner (2002) 
caminham na mesma direção e também utilizam o aparelho metodológico da psicologia, ao procurarem tornar mais claro o papel das emoções na satisfação do consumidor. Os autores utilizam dois experimentos realizados em laboratório ao procurarem confirmar a hipótese de que a satisfação dos consumidores depende tanto de suas expectativas afetivas anteriores ao consumo quanto das emoções vividas durante o consumo do produto. A partir do modelo de expectativas desconfirmadas, eles observam a discrepância entre as emoções antecipadas e as vividas. Assim como na metodologia desenvolvida nesse estudo, Bagozzi e Moore (1994) também realizam experimentos com alunos de graduação em busca das suas respostas emotivas a propagandas de serviços públicos.

A abordagem baseada nos paradigmas da psicologia cognitiva se tornou referência constante para vários outros pesquisadores da área, inclusive, para trabalhos desenvolvidos no Brasil, conforme veremos a seguir.

\section{As emoções e a recente produção acadêmica brasileira sobre marketing}

Analisando a produção brasileira na área de pesquisa de marketing que contempla direta ou indiretamente emoções, encontramos vários trabalhos comprometidos com a consolidação da tradição da psicologia cognitiva. Vários estudos apresentam as emoções como diretamente relacionadas à satisfação do consumidor e indiretamente ligadas à intenção de (re)compra (ALMEIDA; NIQUE, 2004; ESPINOZA; NIQUE, 2004; FARIAS; SANTOS, 2000; LARÁN; ROSSI, 2003; PRADO, 2002) ou aos processos avaliativos pós-compra e/ou consumo (ESPINOZA; NIQUE, 2004; MAYER; ÁVILA, 2006; NICOLAO, 2002). Isso confirma a orientação essencialmente pragmática dos estudos.

As propostas metodológicas constantemente assumem as mesmas prerrogativas da psicologia cognitiva e lançam mão de construções baseadas nos princípios da pesquisa desse campo. Costa e Farias (2004), por exemplo, estudam as emoções e a satisfação do consumidor no ambiente da Internet. Para tanto, os autores combinam técnicas qualitativas e quantitativas e, a partir dos dados levantados da análise de conteúdo de entrevistas em profundidade, propõem um survey. Utilizando escalas baseadas nos trabalhos de Izard (1977) e Oliver (1997) os autores aplicam técnicas multivariadas para confirmar a influência das emoções na satisfação do consumidor on-line.

Lobato, Borges e Nique (2003) procuram validar a escala desenvolvida por Richins (1997), com o intuito de conseguir medir as emoções em experiências de consumo. Lourenço e Rossi (2006) apresentam as emoções como resultantes do envolvimento com produtos de limpeza. A visão das emoções como subproduto de consumo também é levantada por Costa e Larán (2003); DeToni e Schuler (2003); Fonseca, Neto e Gouveia (2003); Silva (2004); Espinoza e Zilles (2004) e por Souza, (2002).

Espinoza e Nique (2003) realizam um levantamento qualitativo das emoções usando a base teórica de Oliver (1993, 1997). Os autores utilizam entrevistas em profundidade com o objetivo de identificar os antecedentes cognitivos e situacionais que levam às emoções em experiências de consumo. Os autores apontam oito proposições de pesquisa - centradas em emoções positivas e negativas e testadas como hipóteses em Espinoza e Nique (2004). Apesar de utilizar ferramental metodológico distinto dos demais trabalhos publicados no Brasil, esse estudo continua comprometido com o arcabouço teórico da psicologia cognitiva e o preceito da capacidade de predição do comportamento do consumidor.

De forma geral, a pesquisa sobre emoções no consumo desenvolvida no Brasil não apresenta desafios que exijam o uso de soluções metodológicas criativas. Na verdade, na maior parte dos estudos, a preocupação com a construção de um conceito de emoção no consumo fica em segundo plano, diante da busca por implicações gerenciais baseadas na observação do comportamento dos consumidores. Mesmo quando as emoções são definidas como "respostas aos resultados das interações sociais" (CARVALHO; VERGARA, 2000, p.4) - o que demonstra uma visão diferente da abordagem cognitiva usual -, o conceito emoção ainda é tratado de forma secundária. 
Outra visão comum a respeito da emoção no consumo é a dicotomia emoção/cognição (FARIAS et alii, 2001; LOBATO; BORGES; NIQUE, 2003) que, a rigor, é utilizada para que os pesquisadores possam reduzir as variáveis envolvidas no estudo. Também nesses casos fica evidente a relativamente pequena preocupação em se explorar as emoções no consumo de um ponto de vista menos operacional. A dedicação às técnicas de tratamento de dados parece mais importante do que o aprofundamento sobre os conceitos.

A emoção também é vista por alguns autores como uma função biológica do sistema nervoso e como força que, juntamente com a razão, participa do processo decisório do consumidor. Nos últimos anos, alguns trabalhos sobre emoções específicas, como o arrependimento (NICOLAO; ROSSI, 2003), vêm surgindo e confirmando o interesse crescente pelo assunto.

Conforme pudemos observar nas seções anteriores, o ponto de vista da psicologia cognitiva domina o cenário de pesquisa sobre emoções no consumo, na medida em que apresenta um ferramental de captura e análise de dados mais desenvolvido e mais alinhado ao paradigma positivista. Certamente, essa visão é importante, uma vez que esses estudos podem servir de orientação para as decisões gerenciais de marketing, mas não podemos deixar de observar, contudo, que os indivíduos vivenciam as emoções de forma muito mais complexa (DENZIN, 1983; HOLBROOK, 1995). Se desejamos conhecer mais sobre os processos emocionais que se desenvolvem em ações de consumo, temos de superar essa perspectiva e procurar outras formas de entendimento do fenômeno emocional. Sob o rótulo de sentimentos, uma ampla gama de aspectos emocionais se desenvolve dentro da mente dos indivíduos (DIENER; LUCAS, 2004) e não deveríamos resumir a pesquisa sobre emoções no consumo à abordagem prática da pesquisa gerencial em marketing.

\section{Perspectivas críticas da pesquisa sobre emoção no comportamento do consumidor}

Conforme vimos, uma das limitações da abordagem tradicional ao processo de decisão de compra é a proposição de que a escolha seria eminentemente determinada por processos basicamente racionais e individuais iniciados pelo reconhecimento de necessidades não satisfeitas e pela procura de informação por parte dos consumidores. Visto por esse prisma, o comportamento do consumidor ganha certa previsibilidade ao se concentrar em ações eminentemente racionais; afinal, as variáveis controladas na pesquisa se tornam mais adequadas ao controle experimental. Da mesma forma, ao se concentrar no indivíduo, a operacionalização da pesquisa também se torna consideravelmente mais simples, na medida em que os pesquisadores podem ser mais facilmente treinados para a coleta e o tratamento dos dados.

A abordagem do comportamento do consumidor baseada na psicologia cognitiva, consolidada na maior parte dos manuais de comportamento do consumidor, não tem mostrado a preocupação de explicitar o processo de construção de significados e valores de consumo. Mesmo que alguns autores considerem o processo emocional posterior ao consumo (OLIVER, 1980; WESTBROOK; OLIVER, 1991), parece inegável a influência (quando não a preponderância) das emoções no processo de construção de significados de consumo (DENZIN, 1984; HOLBROOK, 1986; SEAMON, 1984).

É dentro desse quadro que surgem as primeiras posições críticas com relação ao que está sendo desenvolvido nas pesquisas sobre o comportamento do consumidor. Zajonc (1980), ainda preso ao paradigma da psicologia cognitiva, apresentou outro olhar sobre o processo de formação das preferências: os processos cognitivo e afetivo constituem dois sistemas separados e independentes, de forma que as respostas afetivas não ocorrem necessariamente em seguida à cognição; pelo menos, não em um nível consciente (ZAJONC; MARKUS, 1982). O desencanto com o modelo de representação das emoções a partir das relações entre cognição, afeto e comportamento trouxe alternativas ao estudo do comportamento do consumidor. Conforme proposto por Holbrook (1986), o papel das emoções nas vidas dos consumidores é mais extenso do que havia sido proposto pela abordagem cognitivista. Os aspectos emocionais presentes na experiência de consumo passaram a ser observados de forma mais ampla a partir do modelo proposto por esse autor. 
Partindo dos estudos sobre fantasias, sentimentos e diversão, Holbrook e Hirschman (1982) desenvolveram uma visão alternativa à abordagem cognitivista da emoção no consumo. O modelo proposto envolve consciência, emoção e valor para a experiência de consumo e espera representar de forma mais ampla a natureza do comportamento do consumidor. Essa visão expandida é capaz de revelar a emoção como um ponto de sustentação da experiência de consumo no sentido: consciência - emoção - valor.

A conceptualização de Holbrook (1986) coloca a emoção como a ligação central de um sistema de interação de componentes que compreende um processo múltiplo e dinâmico. Seguindo a proposta de Mehrabian e Russell (1974), Holbrook (1986) distingue entradas pessoais e entradas do ambiente, mas adiciona o terceiro tipo de entrada advindo da interação entre a pessoa e o ambiente. As entradas pessoais compreendem as características gerais do consumidor - tais como características demográficas, socioeconômicas e psicográficas - e os seus recursos, como tempo, energia e dinheiro (HOLBROOK; HIRSCHMAN, 1982). As entradas ambientais apresentam o contraste entre significado, objeto e signo, a unidade simbólica utilizada para designar esse objeto. Nesse modelo, a consciência do consumidor inclui não apenas as crenças sobre atributos de produtos, como no modelo cognitivista convencional, mas também uma variedade de fantasias, sonhos, pensamentos subconscientes e processos mentais inconscientes (HOLBROOK; HIRSCHMAN, 1982).

A definição operacional de consciência é qualquer reação verbal ou não-verbal à entrada de informação da pessoa, do ambiente ou da interação entre pessoa e ambiente. Como conceptualizado por Holbrook (1986), a consciência precede e parcialmente determina a emoção dentro da experiência de consumo. Há uma relação de dependência entre emoção e consciência, mas fica aparente o quanto as emoções podem afetar os processos perceptivos e a atenção que sustentam os estados de consciência.

Ao optar por observar as emoções a partir do referencial da psicologia cognitiva, o pesquisador assume que o objeto de seu estudo encontra-se na mente do indivíduo. O estudo da emoção no marketing parece ver a realidade unicamente com os olhos da psicologia cognitiva. Entretanto, outras disciplinas já se lançaram sobre o estudo das emoções e podem trazer contribuições para o estudo das emoções no consumo. As emoções devem ser vistas não só como expressões de processos internos (micro), mas como complexos multidimensionais (pensamento, sentimento e ação) ou modos de comunicação culturais incorporados que surgem de relações de poder e interdependência (macro) (STURDY, 2003).

As emoções seriam inimagináveis sem, pelo menos, a presença de outro indivíduo, como já expôs Sartre (1965), além de Hochschild (1979) e Denzin (1983), os dois últimos representantes do campo da sociologia das emoções. Mesmo vivenciadas internamente, as emoções são fundamentalmente interacionais, e essas interações podem acontecer entre uma pessoa e quaisquer entidades, concretas ou imaginárias. O consumidor está envolvido em uma teia social que impede ou produz determinadas ações emocionais diante das suas experiências de consumo. De forma reflexiva, suas experiências durante o consumo também influenciam suas emoções. Dessa forma, nos parece evidente a necessidade de se observar outros paradigmas que complementem o conhecimento do campo do comportamento do consumidor.

\section{Bases sociais das emoções}

Apesar de suas bases biológicas, as emoções incorporam uma grande parte do aprendizado que ocorre socialmente e que é moldado pela sociedade (NUSSBAUM, 1997). O medo, por exemplo, não é constituído apenas pela sensação de frio na barriga, mas também envolve considerações sobre o perigo e sobre a origem e potencial desse perigo. Mesmo que alguns estudos atribuam um caráter universal a certas manifestações emocionais, através de expressões faciais e/ou corporais (EKMAN; FRIESEN, 1986), para outros autores as emoções não são apenas fenômenos biopsicológicos inatos. Alguns estudos históricos e culturais mostram diferenças entre fenômenos emocionais experimentados por indivíduos, as quais estão / estariam relacionadas a significados, à demonstração e à regulação das emoções (LUTZ, 1988; HOCHSCHILD, 1979). 
O campo da sociologia das emoções está focado no exame das circunstâncias sociais que elicitam emoções ou sentimentos específicos e se dedica ao estudo dos antecedentes sociais das emoções a partir de dois níveis: do micro (que parte do indivíduo e é constantemente associado à psicologia social) e do macro (que parte da sociedade e é associado à perspectiva estrutural e cultural).

\section{Abordagem das emoções em nível micro}

Os estudos que analisam o fenômeno emocional a partir do indivíduo são constantemente associados à psicologia social. Por certo, a psicologia social serve como "ponte" entre a psicologia e a sociologia e, portanto, tornou-se a primeira opção de estudo das influências sociais sobre as emoções.

Os pesquisadores mais alinhados à perspectiva da psicologia social, chamados de "positivistas" (aqui entre aspas conforme a discussão original da sociologia), tratam a emoção como variável dependente e aceitam o argumento da "especificidade" (correspondências existentes entre componentes das emoções um a um) (KEMPER, 1981). Dessa forma, para esses pesquisadores, certas classes de estímulos ambientais levam a respostas autônomas específicas, a comportamentos expressivos específicos e, então, a emoções específicas. Os eventos sociais são, dessa forma, os determinantes das emoções. Kemper (1981), por exemplo, assume que certas classes de eventos situacionais (decrementos de poder e status, mais notadamente) estimulam direta e universalmente a mesma emoção porque as ligações entre eventos e emoções têm base psicológica. Os "positivistas" consideram as emoções respostas automáticas e padronizadas conforme classes particulares de estímulos sociais (KEMPER, 1981; SCHEFF, 1990).

Em contrapartida, as perspectivas "culturalistas" assumem as emoções como relacionadas à situação e aos vocabulários e crenças emocionais, que variam de acordo com o tempo e a localização do indivíduo. A mesma classe de eventos situacionais podem ser interpretada como emoções diferentes em culturas diferentes, a despeito de suas bases psicológicas. Dessa perspectiva não faz muito sentido pensarmos em variáveis dependentes ou independentes, porque, dadas as construções múltiplas e constantes de vocabulários emocionais dentro dos grupos sociais, torna-se inadequado separarmos as emoções dos eventos sociais, propriamente. O próprio acervo de emoções do indivíduo o leva a perceber o evento social de maneira própria não fazendo sentido estabelecer aí uma relação de causa e efeito.

Contudo, as abordagens interacionistas e construcionistas, que compõem a perspectiva sociocultural, não são propriamente idênticas. Os construtivistas sociais se aproximam da perspectiva macro e focam na demonstração do grau em que emoções - tanto básicas (medo, surpresa, raiva, nojo, tristeza, felicidade e contentamento), quanto não-básicas (vergonha, culpa, amor, ansiedade e ressentimento, entre outras) - são constituídas ou influenciadas social e culturalmente. Por sua vez, os interacionistas simbólicos assumem que os eventos ambientais possibilitam o estímulo geral, interpretado como uma emoção particular com base nos fatores situacionais que mais se sobressaiam. As emoções são o produto da junção do estímulo geral e de fatores socioculturais específicos - definições da situação e rótulos culturais. O mesmo estímulo pode ser experienciado como felicidade ou raiva, dependendo das pistas situacionais disponíveis.

Uma vez que definições situacionais e rótulos emocionais variam no tempo e entre culturas, também variam as experiências emocionais, de acordo tanto com os construtivistas sociais, quanto com os interacionistas simbólicos. Os determinantes da experiência emocional não são fisiológicos, mas socioculturais. Existem tantas emoções quantas são distinguidas em uma cultura e entre culturas.

Uma visão integradora entre as perspectivas "positivista" e sociocultural foi proposta por Kemper (1987). Ele sugere que as emoções básicas (ou primárias), de origem fisiológica, tornam-se elaboradas a partir de rótulos, significados e definições sociais relacionados a diferentes condições de interação e de organização social. Já as emoções secundárias (ou não-básicas) seriam adquiridas a partir da junção de experiências emocionais primárias do indivíduo com os rótulos secundários associados a situações específicas. Os agentes socializadores teriam o papel de rotular as experiências emocionais dos indivíduos, elicitando os sentimentos ou sensações 
primárias. Essa visão é mais balanceada e permite um diálogo maior entre os campos da psicologia e da sociologia, que pode ser interessante para a pesquisa em marketing, pois pode se utilizar todo o acervo metodológico desenvolvido em pesquisa específica sobre consumo.

\section{Abordagem das emoções em nível macro}

Se formos observar, cronologicamente, o desenvolvimento do interesse da sociologia pelas emoções começou com abordagens eminentemente macro. Como destaca Scheff (2001), um dos primeiros autores de sociologia a dar importância aos estados emocionais de indivíduos na sociedade foi Norbert Elias. Ao realizar uma retrospectiva histórica dos costumes a partir de manuais de aconselhamento, Elias (1990) sugere que um dos aspectos centrais da modernidade é o sentimento de vergonha e apresenta uma proposta de transmissão social da vergonha e dos tabus.

Outros autores da sociologia têm reconhecida importância como antecessores da escola da sociologia das emoções: Sennett, Cobb e Lynd. Assim como Elias, Sennett e Cobb (1972) também utilizaram o sentimento de vergonha como importante componente de compreensão e análise do processo de construção social, a partir de estudos conduzidos em escolas norte-americanas. Lynd (1961) aproximou-se do sentimento de vergonha com o objetivo de entender o processo de construção da identidade dos indivíduos, ressentindo-se, entretanto, de ferramentas metodológicas que lhe possibilitassem um conhecimento mais aprofundado.

A quantidade de estudos macro sobre as emoções ainda é pequena, dadas as dificuldades inerentes a esse tipo de estudo. Estudos sobre a intensidade de tipos particulares de emoções em populações ou sobre mudanças na cultura emocional partem dos resultados e das características populacionais. Tais estudos prescindem da capacidade de construção da análise de um corpus de dados muito amplo.

\section{Implicações para o estudo das emoções no marketing}

Bagozzi, Gürhan-Canli e Priester (2002) sugerem que os pesquisadores de marketing interessados em ampliar o conhecimento a respeito do papel das emoções no consumo devem partir para o estudo das bases sociais das emoções. Um amplo acervo de experiências emocionais surge de respostas advindas do grupo social no qual o indivíduo se insere e das interpretações dessas respostas. Sendo o consumo uma atividade eminentemente social, essa abordagem pode contribuir de forma significativa para se conhecer melhor o comportamento dos consumidores.

Ao admitirmos múltiplas abordagens, capacitamo-nos a perceber o mundo das emoções e do consumo em diversas frentes, ampliando as possibilidades de se desenvolver conhecimento relevante sobre o papel dessas emoções no consumo. A forma pela qual o indivíduo é ensinado a se comportar emocionalmente, assim como a forma pela qual ele interpreta sinais sociais, pode nos levar a compreender determinados comportamentos de consumo e suas variações. Da mesma forma, poderemos observar e entender mais claramente as propostas de interações entre consumidores, produtos, produtores e marcas construídas e interpretadas dentro de um mundo social, tornando mais evidentes as intenções dos consumidores. Surgiriam, então, formas de se entender o comportamento do consumidor e as variações desse comportamento, que possam subsidiar ações empresariais mais afinadas com os desejos dos consumidores.

O estudo dos antecedentes sociais do comportamento do consumidor pode oferecer subsídios para uma compreensão mais aprofundada do processo de valoração de bens e serviços. Ao entendermos como o consumidor constrói sua teia de relações de consumo e como o vocabulário emocional do indivíduo é aprendido, poderemos oferecer aos praticantes novas opções de atuação. As estratégias de comunicação poderão ser mais eficientes ao buscar "emocionar" o consumidor ou, ainda, ir ao encontro de suas emoções a partir dos significados construídos. Diversas linhas de produtos e serviços poderão ser desenvolvidas para atender às "demandas emocionais". Devido ao suporte teórico e metodológico mais próximo do universo do sujeito investigado, a compreensão do seu estado emocional não será mais calcada numa lista de possibilidades, 
mas, sim, de relatos historicamente fundamentados na vida desse sujeito. Tal demanda emocional, portanto, será compreendida mais naturalmente, possibilitando que seja descrita de forma mais detalhada e sugestiva.

Da mesma forma, poderemos propor instrumentos de proteção ao consumidor e às outras entidades envolvidas com os processos de troca e suas conseqüências. Ao entendermos como as emoções interferem na construção de significados de consumo dos indivíduos, tornamos a questão mais clara não apenas para os que lidam com marketing, mas também para os consumidores e para outras instituições interessadas em conhecer o comportamento dos mercados (espaços de encontro entre empresas e consumidores). Entre essas outras instituições podemos incluir governos ou quaisquer interessados nos movimentos ocorridos na arena do mercado.

Outra aplicação possível - paralelamente à realização de novas pesquisas - inclui o desenvolvimento de políticas públicas e a atuação governamental em algumas áreas críticas da saúde pública, como a que trata da prevenção de doenças sexualmente transmissíveis e do uso de drogas. Em relação a essas duas questões, por exemplo, um melhor entendimento sobre a influência das emoções no consumo pode ser extremamente proveitoso na elaboração de campanhas de conscientização a respeito da proteção necessária para a prática de sexo seguro e de prevenção de problemas relacionados ao uso de drogas.

Evidentemente, a implementação e o amadurecimento dessas propostas dependem de uma disposição maior por parte dos pesquisadores de marketing e de comportamento do consumidor em adotar métodos de pesquisa qualitativos, por vezes, mais "exploratórios" que "conclusivos". Boa parte desse conhecimento requer investigação de caráter indutivo que, ao invés de confirmar teorias importadas, ajude a desenvolver teorias que melhor se ajustem aos diversos ambientes culturais do Brasil. Isso se aplica tanto aos pesquisadores no campo da academia quanto aos que se dedicam à pesquisa gerencial de mercado. Afinal, teorias formuladas por acadêmicos são fundamentais para a boa representação dos diferentes consumidores brasileiros e, portanto, de valor inestimável para pesquisadores de mercado e para empresas que atendam a esses consumidores tão mal representados. 


\section{Referências}

AACKER, D. A.; STAYMAN, D. M.; HAGERTY, M. R. Warmth in advertising: measurement, impact and sequence effects. Journal of Consumer Research, v.12, n.4, p.365-382, 1986.

AHTOLA, O. T. Hedonic utilitarism aspects of consumer behavior: an attitudinal perspective. Advances in Consumer Research, v.12, p.7-10, 1985.

AHUVIA, A. C. Beyond the extended self: loved objects and consumers' identity narratives. The Journal of Consumer Research, v.32, p.171-184, 2005.

ALMEIDA, S.O.; NIQUE, W.M. Encantamento do cliente: compreendendo o conceito e validando o constructo. In: ENCONTRO NACIONAL DOS PROGRAMAS DE PÓS-GRADUAÇÃO EM ADMINISTRAÇÃO, 28., 2004, Curitiba. Anais... Curitiba: Anpad, 2004. CD-ROM.

BAGOZZI, R. P.; GOPINATH, M.; NYER, P. U. The role of emotions in marketing. The Journal of Academy of Marketing Science, v.27, p.184-206, 1999.

; GÜRHAN-CANLI, Z. PRIESTER, J. R. The social psychology of consumer behavior. Buckingham: Open University Press, 2002.

; MOORE, D. J. Public service advertisements: emotions and empathy guide pro-social behavior. Journal of Marketing, v.58, p.56-70, 1994.

BATRA, R. Understanding the likeability/involvement interaction: the 'overide' model. Advances in Consumer Research, v.12, p.362-367, 1985.

; SILK, A. Recall, recognition and the measurement of memory for print advertisements. Marketing Science, v.2, p.95-134, 1984.

BENTON JR., R. Work, consumption and the joyless consumer. IN: FIRAT, A. F.; DHOLAKIA, N.; BAGOZZI, R. P. Philosophical and radical thought in marketing. New York: Rowman \& Littlefield, 1987.

CARVALHO, J. L. F. S.; VERGARA, S. C. Repensando os roteiros de marketing de serviços - análise crítica da teoria dos scripts cognitivos. ENCONTRO NACIONAL DOS PROGRAMAS DE PÓS-GRADUAÇÃO EM ADMINISTRAÇÃO, 24., 2000, Florianópolis. Anais... Florianópolis: Anpad, 2000. CD-ROM.

COSTA, A. C. R.; FARIAS, S. A. Emoções e satisfação em compras on-line: o "ser" é humano em ambientes intermediados por computadores? In: ENCONTRO NACIONAL DOS PROGRAMAS DE PÓS-GRADUAÇÃO EM ADMINISTRAÇÃO, 28., 2004, Curitiba. Anais... Curitiba: Anpad, 2004. CD-ROM.

COSTA, F. C. X.; LARÁN, J. A. Influências ambientais e compra por impulso: um estudo em lojas virtuais e físicas. In: ENCONTRO NACIONAL DOS PROGRAMAS DE PÓS-GRADUAÇÃO EM ADMINISTRAÇÃO, 27., 2003, Atibaia. Anais... Atibaia: Anpad, 2003. CD-ROM.

DENZIN, N. K. A note on emotionality, self and interaction. The American Journal of Sociology, v.89, n.2, p.402-409. 1983.

On understanding emotion. San Francisco: Jossey-Bass, 1984.

DETONI, D.; SCHULER, M. Imagem de produto e comportamento do consumidor: explorando o processo de formação de imagens. In: ENCONTRO NACIONAL DOS PROGRAMAS DE PÓS-GRADUAÇÃO EM ADMINISTRAÇÃO, 27., 2003, Atibaia. Anais... Atibaia: Anpad, 2003. CD-ROM.

DIENER, E.; LUCAS, R. E. Subjective emotional well-being. In: LEWIS, M.; HAVILAND-JONES, J. M. Handbook of emotions. 2.ed. New York: Guilford Press, 2004. 
EKMAN, P.; FRIESEN, W. V. A new pan-cultural facial expression of emotion. Motivation \& Emotion, v.10, p.159-168, 1986.

ELIAS, N. O processo civilizador: uma história dos costumes. Rio de Janeiro: J. Zahar, 1990.

ENGEL, J. F.; BLACKWELL, R. D.; MINIARD, P. W. Comportamento do consumidor. 8.ed. Rio de Janeiro, LTC, 2000.

ESPINOZA, F. S.; NIQUE. W. M. Experiências emocionais em situações de consumo de produtos: evidências e proposições de pesquisa. In: ENCONTRO NACIONAL DOS PROGRAMAS DE PÓS-GRADUAÇÃO EM ADMINISTRAÇÃO, 27., 2003, Atibaia. Anais... Atibaia: Anpad, 2003. CD-ROM.

O impacto de experiências emocionais na atitude e intenção de compra do consumidor: o papel da relevância e da congruência com os objetivos pessoais. In: ENCONTRO NACIONAL DOS PROGRAMAS DE PÓS-GRADUAÇÃO EM ADMINISTRAÇÃO, 28., 2004, Curitiba. Anais... Curitiba: Anpad, 2004. CD-ROM.

; ZILLES, F. A geração de afeto negativo a partir da atmosfera da loja e sua influência na intenção de retorno e recomendação do consumidor In: ENCONTRO NACIONAL DOS PROGRAMAS DE PÓS-GRADUAÇÃO EM ADMINISTRAÇÃO, 28., 2004, Curitiba. Anais... Curitiba: Anpad, 2004. CD-ROM.

FARIAS, S. A. et al. O comportamento de presentear: dimensões motivacionais relevantes para o marketing. In: ENCONTRO NACIONAL DOS PROGRAMAS DE PÓS-GRADUAÇÃO EM ADMINISTRAÇÃO, 25., 2001, Campinas. Anais... Campinas: Anpad, 2001. CD-ROM.

FARIAS, S. A.; SANTOS, R. C. Influência da resposta afetiva na satisfação do consumidor: um estudo no segmento da terceira idade. In: ENCONTRO NACIONAL DOS PROGRAMAS DE PÓS-GRADUAÇÃO EM ADMINISTRAÇÃO, 24., 2000, Florianópolis. Anais... Florianópolis: Anpad, 2000. CD-ROM.

FISHBEIN, M.; AZJEN, I. Belief, attitude, intention and behavior: an introduction to theory and research. New York: Addison-Wesley Pub. Co. Inc., 1979.

FONSECA, F. R. B.; NETO, A. F. S.; GOUVEIA, T. B. Acessando a reação emocional à propaganda: um estudo baseado na escala de William D. Wells. In: ENCONTRO NACIONAL DOS PROGRAMAS DE PÓS-GRADUAÇÃO EM ADMINISTRAÇÃO, 28., 2003, Atibaia. Anais... Atibaia: Anpad, 2003. CD-ROM.

FOURNIER, S. Consumers and their brands: developing relationship theory in consumer research. The Journal of Consumer Research, v.24, n.4, p.343-373, 1998.

FRIJDA, N. H. The psychologists' point of view. In: LEWIS, M.; HAVILAND-JONES, J. M. Handbook of Emotions. 2.ed. New York: Guilford Press, 2004.

HIRSCHMAN, E. C.; STERN, B. The roles of emotion in consumer research. Advances in Consumer Research, v.26, p.1$11,1999$.

HOCHSCHILD, A. Emotion work, feeling rules and social structures. American Journal of Sociology, v.85, p.551-575, 1979.

HOLBROOK. M. B. Emotion in consumption experience: toward a new model of the human consumer. In: PETERSON, R. A.; HOYER, W. D.; WILSON, W. R. The role of affect in consumer behavior. Massachusetts: Lexington Books, 1986.

Consumer research - introspective essays on the study of consumption. Thousand Oaks: Sage, 1995.

BATRA, R. Assessing the role of emotions as mediators of consumer responses to advertising. Journal of Consumer Research, v.14, p.404-420, 1987.

; HIRSCHMAN, E. C. The experiential aspects of consumption, feelings and fun. Journal of Consumer Research. V.9, p.132-140, 1982. 
HOWARD, J. A. Mercadologia: comportamento do administrador e do comprador. Rio de Janeiro: J. Zahar, 1969.

IZARD, C. Human emotions. New York: Plenum, 1977.

KEMPER, T. D. Social constructivist and positivist approaches to the sociology of emotions. American Journal of Sociology, v.87, p.336-362, 1981.

. How many emotions are there? Wedding the social and autonomic components. American Journal of Sociology, v.93, p.263-289, 1987.

LARÁN, J. A.; ROSSI, C. A. V. O poder da surpresa no processo emocional de formação da satisfação. In: ENCONTRO NACIONAL DOS PROGRAMAS DE PÓS-GRADUAÇÃO EM ADMINISTRAÇÃO, 27., 2003, Atibaia. Anais... Atibaia: Anpad, 2003. CD-ROM.

LAZARUS, R. S. Thoughts on the relations between emotion and cognition. American Psychologist, v.37, p.1019-1024, 1982.

LOBATO, A.; BORGES, A.; NIQUE, W. M. Avaliação da aplicabilidade da escala consumption emotions set para mensuração das emoções em experiências de consumo na cidade de Porto Alegre. In: ENCONTRO NACIONAL DOS PROGRAMAS DE PÓS-GRADUAÇÃO EM ADMINISTRAÇÃO, 27., 2003, Atibaia. Anais... Atibaia: Anpad, 2003. CD-ROM.

LOURENÇO, E. C.; ROSSI, G. B. Sentimentos e envolvimento diferenciando marcas: um estudo em supermercados na cidade de Campinas/SP. In: ENCONTRO NACIONAL DOS PROGRAMAS DE PÓS-GRADUAÇÃO EM ADMINISTRAÇÃO, 30., 2006., Salvador. Anais... Salvador: Anpad, 2006. CD-ROM.

LUCE, M. F., PAYNE, J. W.; BETTMAN, J. R., Emotional trade-off difficulty and choice. Journal of Marketing Research, v.36, p.143-159, 1999.

LUTZ, C. A. Unnatural emotions: everyday sentiments on a Micronesian atoll and their challenge to Western theory. Chicago: University of Chicago Press, 1988.

LUTZ, R. J.; MACKENZIE, S. B.; BELCH, G. E. Attitude toward the ad as a mediator of advertising effectiveness: determinants and consequences. Advances in Consumer Research, v.10. p.532-539, 1983.

LYND, H. M. On shame and the search for identity. New York: Science Editions, 1961.

MAYER, V. F.; ÁVILA, M. Percepções de injustiça em aumentos de preços de serviços: um estudo experimental sobre dependência do consumidor, relevância do serviço, emoções e intenções de comportamento. In: ENCONTRO NACIONAL DOS PROGRAMAS DE PÓS-GRADUAÇÃO EM ADMINISTRAÇÃO, 30., 2006, Salvador. Anais... Salvador: Anpad, 2006. CDROM.

MEHRABIAN, A.; RUSSELL, J. A. An approach to environmental psychology. Cambridge: MIT Press, 1974.

MOORE, B. E.; FINE, B. D. Termos e conceitos psicanalíticos. Porto Alegre: Artes Médicas, 1992.

MOORMAN, C. Marketing as technique: the influence of marketing on the meanings of consumption. In: FIRAT, A. F.; DHOLAKIA, N.; BAGOZZI, R. P. Philosophical and radical thought in marketing. Massachusetts: Lexington, 1987.

NICOLAO, L. Compreendendo o arrependimento no processo de tomada de decisão do consumidor. In: ENCONTRO NACIONAL DOS PROGRAMAS DE PÓS-GRADUAÇÃO EM ADMINISTRAÇÃO, 26., 2002, Salvador. Anais ... Salvador: ANPAD, 2002. CD-ROM.

; ROSSI, C.A.V. Desenvolvimento e validação de uma escala de arrependimento no processo de tomada de decisão do consumidor. In: ENCONTRO NACIONAL DOS PROGRAMAS DE PÓS-GRADUAÇÃO EM ADMINISTRAÇÃO, 27., 2003, Atibaia. Anais... Atibaia: Anpad, 2003. CD-ROM.

NUSSBAUM, M.C. Cultivating humanity. Cambridge: Harvard University Press, 1997. 
OLIVER, R. L. A cognitive model of the antecedents and consequences of satisfaction decisions. Journal of Marketing Research, v.17, p.460-69, 1980.

. Cognitive, affective, and attribute bases of the satisfaction response. Journal of Consumer Research, v.20, p.418-430, 1993.

Satisfaction: a behavioral perspective on the consumer. Boston: Irwin McGraw-Hill, 1997.

PARKINSON, B. Ideas and realities of emotion. London: Routledge, 1995.

PETERSON, R. A.; HOYER, W. D.; WILSON, W. R. Reflections on the role of affect in consumer behavior In: PETERSON, R. A.; HOYER, W. D.; WILSON, W. R. The role of affect in consumer behavior. Massachusetts: Lexington Books, 1986.

PHILLIPS, D. M.; BAUMGARTNER, $H$. The role of consumption in the satisfaction response. Journal of Consumer Psychology, v.12, n.3, p.243-252, 2002.

PLUTCHIK, R. Emotion: a psychoevolutionary synthesis. New York: Harper \& Row, 1980.

PRADO, P. H. M. Integração da qualidade percebida, resposta afetiva e satisfação no processo de compra/consumo de serviços. In: ENCONTRO NACIONAL DOS PROGRAMAS DE PÓS-GRADUAÇÃO EM ADMINISTRAÇÃO, 26., 2002, Salvador. Anais... Salvador: Anpad, 2002. CD-ROM.

RICHINS, M. L. Measuring emotions in the consumption experience. Journal of Consumer Research, v.24, p.127-146, 1997.

SARTRE, J. P. Esboço de uma teoria das emoções. Rio de Janeiro: Zahar, 1965.

SCHEFF, T. J. Microsociology: discourse, emotion and social structure. Chicago: The University of Chicago Press, 1990.

. Três pioneiros da sociologia das emoções. Política \& Trabalho, v.17, p.115-127, 2001.

SEAMON, D. Emotional experience. American Behavioral Scientist, v.27, p.337-347, 1984.

SENNETT, R.; COBB, J. The hidden injuries of class. New York: Vintage Books. 1972.

SILVA, P. G. A cultura é importante para o consumidor e para o marketing? Investigando a "dança" do patrocínio e o "ritmo" do envolvimento. In: ENCONTRO NACIONAL DOS PROGRAMAS DE PÓS-GRADUAÇÃO EM ADMINISTRAÇÃO, 28., 2004, Curitiba. Anais... Curitiba: Anpad, 2004. CD-ROM.

SOUZA, R.V. Prontidão para tecnologia do consumidor: aspectos emocionais da adoção de produtos e serviços baseados em tecnologia. In: ENCONTRO NACIONAL DOS PROGRAMAS DE PÓS-GRADUAÇÃO EM ADMINISTRAÇÃO, 26., 2002, Salvador. Anais... Salvador: Anpad, 2002. CD-ROM.

STURDY, A. Knowing the unknowable? A discussion of methodological and theoretical issues in emotion research and organizational studies. Organization, v.10, n.1, p.81-105, 2003.

TSAL, Y. On the relationship between cognitive and affective processes: a critique of Zajonc and Markus. Journal of Consumer Research, v.12, p.358-362, 1985.

WESTBROOK, R. A.; OLIVER, R. L. The dimensions of consumption emotion patterns and consumer satisfaction. Journal of Consumer Research, v.18, p.84-91, 1991.

ZAJONC, R. B. Feeling and thinking: preferences need no inferences. American Psycholgist, v.35, n.2, p.151-175, 1980. ; MARKUS, H. Affective and cognitive factors in preferences. Journal of Consumer Research, v.9, p.123-131,

1982.

; Must all be mediated by cognition? Journal of Consumer Research, v.12, p.363-364, 1985. 